\title{
Living Donor Liver Transplantation for Hepatocellular Carcinoma
}

\author{
Chih-Che Lin, \\ Ahmed Mohammed Abdel Aziz Elsarawy and \\ Chao-Long Chen
}

Additional information is available at the end of the chapter

http://dx.doi.org/10.5772/65109

\begin{abstract}
Hepatocellular carcinoma (HCC) is a major worldwide health problem, which is expected to increase steadily due to different underlying liver diseases. Surgical treatment modalities including liver transplantation (LT) or liver resection (LR) are the mainstay options for early cases of HCC. Liver transplantation for well-selected cases provides excellent survival outcomes comparable to nonmalignant indications of LT. Living donor liver transplantation (LDLT) is an alternative option or even the sole one in the current era of organ shortage problem and in some Asian countries where deceased organ donation is markedly reduced due to various reasons. The adoption of LDLT for HCC treatment elicited many dynamic changes and debates to the dilemma of LT as a whole. In this chapter, we focus on different perspectives of LDLT for HCC, including selection criteria evolution, controversial topics, ethical considerations, operative highlights, and other points.
\end{abstract}

Keywords: hepatocellular carcinoma, living donor, liver, transplantation, criteria, loco-regional therapy

\section{Introduction}

Hepatocellular carcinoma (HCC) is the sixth most common cancer and the third leading cause of deaths globally. The selection of treatment modalities for HCC is challenging because it depends not only on the stage of tumor and the patient's performance but also on the underlying liver function. Though the staging systems of HCC are clinically useful for 
treatment allocation, the decision making should be tailored to each patient, taking into account morphological, pathological, and biological tumor criteria. Liver resection(LR) or liver transplantation (LT) can be adopted as a surgical curative therapy for early disease. Ablative therapies such as radiofrequency ablation (RFA) and percutaneous ethanol injection (PEI) can also cure small tumors. Transarterial chemoembolization (TACE), transarterial radio embolization (TARE), and external beam radiation therapy (EBRT) can control locally advanced disease that is no longer amenable to cure [1].

Liver transplantation has been considered theoretically the best treatment for HCC since it cures the cancer and its underlying pathology. Milan criteria (MC) were the cornerstone for LT because patient selection according to them resulted in survival outcome comparable to LT for nonmalignant cases. More cases of HCC were subjected to LT with the changes made in 2002 in western countries when additional points were added to the model for end-stage liver disease (MELD) scoring system for HCC patients that are within MC. However, the protracted waiting list for deceased donor liver transplantation (DDLT) took many patients beyond MC and hence lost their chance of LT [2].

The incorporation of living donor liver transplantation (LDLT) into the treatment roadmaps of HCC not only gave a new horizon to more patients, but also elicited many dynamic scientific debates and opened different perspectives for the field of LT in general and the treatment of HCC in particular. Thanks to the extensive research and experience of many groups working on LDLT, especially the Asian transplantation groups, most LT centers now are achieving excellent results.

Specifically to the treatment of HCC, LDLT has several major advantages including: avoidance of prolonged waiting times, allowing elective timing of transplantation under best circumstances both for the patient and the tumor burden, not consuming the public donor pool that may reduce the chance of LT for nonmalignant cases awaiting transplant, and providing organ replacement therapy in parts of the world where brain death and deceased donation are less commonly accepted [3].

In addition to the conventional hepatitis virus-related HCC, a steadily increasing burden of HCC will be witnessed with the emergence of alcohol-related cirrhosis and nonalcoholic steatohepatitis (NASH) as potential major causes of HCC, adding more burden to the transplantation demands and worsening the worldwide problem of organ shortage. It has been recognized that fine-tuning and timing of liver transplantation for HCC are only possible with live organ donation. For these reasons, the need for implementation and optimization of LDLT program will continue in the near future [4-6].

In the following sections, we will discuss basic concepts, criteria evolution, ethical considerations, and controversial topics regarding LDLT for HCC treatment, some of them are commonplace among LDLT and DDLT. The technical details of the procedure of LDLT in general are beyond the scope of this chapter. However, surgical details that are related to the oncologic outcome of the patients with HCC are highlighted. 


\section{Current practice of LDLT for HCC}

HCC represents more than $90 \%$ of primary liver cancers and It has a well-remarked geographic distribution. Around 85\% of cases occur in East Asia, sub-Saharan Africa, and Melanesia. The great burden of HCC is most prevalent among the less developed world. In developed regions, the incidence is low, except for Southern Europe where the incidence is significantly higher [7, 8].

By and large, LDLT has emerged as an alternative to DDLT and it is the only option for patients with HCC in many Asian countries, where the problem of organ shortage is commonly faced. It also provides a suitable option for patients exceeding the MC in western countries such as the United States and Europe, and in the absence of legislative regulations and setup for DDLT in some developing countries especially in the Middle East and North Africa [9-11].

At the beginning of this century, it was estimated that LDLT would represent a significant proportion of the patients transplanted with HCC. Unfortunately, the risks of death and major complications for the healthy donors at the early experience ( 0.3 and $2 \%$, respectively) reduced its practice on a wide scale. With the cumulative experiences over the past two decades, LDLT is now practiced smoothly in centers of excellence with experience of hepatobiliary surgery and transplantation medicine $[7,12]$.

\subsection{Western experience}

Since 2002, HCC patients who are within Milan criteria gain additional points under the MELD organ allocation policy. This resulted in shorter waiting time for HCC patients in many regions of the United States, obviating the need for LDLT. In some regions, however, longer waiting times with higher dropout rates support the use of LDLT for cases of HCC within the Milan criteria and in candidates who do not meet criteria for waiting list priority [13].

The situation of LDLT within the western experience can be configured from the 2012 release of European Association of Study of the Liver (EASL) guidelines, that mentioned "Living donor liver transplantation is an alternative option in patients with a waiting list exceeding 67 months, and offers a suitable setting to explore extended indications within research programs" [7].

In the United States, approximately 7000 new patients with HCC are put on the waiting list for LT every year, $10-15 \%$ of whom die during the waiting period. In Europe and the United States, the dropout rate at various centers ranges between 15 and 35\%. From this perspective, LDLT offer a survival advantage to a significant proportion of HCC patients in the western world [14].

\subsection{Eastern experience}

The liver transplantation setup in Asia differs from that in the West, in the fact that no priority is given to HCC patients. This setup is formulated to avoid the inevitable shift of most of the deceased grafts to the persistently increasing HCC cases on the expense of nonmalignant cases 
waiting LT. The resultant wait-list lead Asian centers to practice various approaches to cover the needs, including bridging treatment for HCC, salvage transplantation after prior hepatectomy and LDLT for HCC. Shortage of donors in Asian countries is attributed to many factors including cultural and religious beliefs [10, 15].

\section{Basic concepts}

\subsection{Philosophy}

The most prominent advantage of LDLT for HCC treatment is the reduction of waiting time on the list for all cases including other etiologies of liver failure with nearly no effect on public donor pool. Living donor liver grafts are dedicated gifts to the recipients and are generally of good quality taking into account that most of living donors are healthy young individuals [15, 16].

The concept of "gifting" allowed LDLT for HCC patients who are beyond MC or any other proposed criteria and opened the door for liberal expansion. This concept is not accepted in the wait list of DDLT in which the procedure is conditioned to the "within criteria" cases only. The concept of "gifting" must not be taken for granted in the case of LDLT. Since the risk of a living donor is taken, selection criteria must be adopted for HCC patients with optimization of the pretransplantation conditions to ensure a high survival outcome. In other words, the readily available willing donor does not justify the ultramajor procedure of transplantation in the absence of a survival benefit for the recipient. In addition, donor safety remains the other pillar of a sound decision of LDLT [17].

\subsection{Indications}

LDLT is indicated in HCC patients, who are within accepted universal or regional criteria for transplantation. In view of organ scarcity, the indications can be described currently to be result-oriented in terms of overall and recurrence-free survival. Consequently, there is marked variation between different regions and institutions for applied indications. In essence, cases fitting Milan criteria are indicated for LDLT if waiting list would be $>7$ months or if there is a willing available donor. Milan criteria form the solid base of morphological indications for HCC. As discussed later, LDLT has allowed transplantation for patients who are outside Milan criteria according to the dynamically changing criteria in different institutions $[18,19]$.

\subsection{Contraindications}

Likewise, there is heterogeneity in the contraindications between different regions according to criteria limits for patient selection. However, there is a body of evidence that LDLT is absolutely contraindicated in the following conditions: cases of HCC that show major vascular invasion as evidenced by pretransplant imaging, the presence of extrahepatic metastases including suspicious porta hepatis nodal disease and cases with ruptured HCC. Absolute 
contraindications of LDLT in nonmalignant cases hold true for HCC and include cases with uncontrolled systemic infection or lethal uncontrolled medical comorbidities [20].

\section{Terminology}

With the implementation of LDLT programs and better understanding of the clinical course and biological nature of HCC, various clinical settings have emerged. In addition, the current progress of the interventional anticancer therapies enforced the armamentarium of the HCC management for all clinical scenarios. The following are different settings rather than different types of LDLT.

\subsection{Primary LDLT}

It is the procedure of LDLT performed upfront for the treatment of the cancer and underlying primary liver disease. In this setting, no previous liver resection is performed but initial ablative loco-regional therapies like RFA or PEI may have been performed. It is carried out for nonresectable HCC cases fitting the standard Milan criteria, UCSF criteria or other established and justified criteria for individual institutions. According to Barcelona Clinic Liver Cancer (BCLC) guidelines, primary LDLT is conducted for early cases of HCC with poor liver functions that cannot tolerate initial resection or ablation [7].

\subsection{Salvage LDLT}

In this setting, LT is performed when there is tumor recurrence or deterioration of liver functions after initial hepatectomy. The integral point in salvage LDLT is that it is performed aiming at a similar outcome to nonrecurrent cases. The pretransplant setting in the case of salvage LT after previous hepatectomy differs from that of primary LT in the availability of tissue pathology, so that almost all factors that determine tumor's behavior (grade, the presence or absence of microvascular invasion, the grade of necrosis in response to loco-regional therapy) are determined beforehand. That is why the predictive power of outcome of salvage LT should be more precise. Very importantly, we should consider that patients who have "within criteria" recurrence after hepatectomy, and hence amenable to salvage LT, are not more than $25 \%$ in some series. This means that nearly $70 \%$ of cases may miss the chance of a curative LT at all. The transplantability at the time of recurrence has been found to be an important variable determining the final outcome [21]. Noteworthy that when salvage LT is considered due to transplantable recurrence, the recurrence pattern should be evaluated before embarking into LT even if the liver functions and tumor burden are amenable to LT. Intrahepatic recurrent tumors after hepatectomy originates from either primary tumor metastases or de novo tumor foci. The interval and histopathological analysis of hepatectomy specimens give a clue to the pattern of recurrence. Tumors recurring in the first 12 months are most likely tumor metastases within the liver, while those arising after 12 months represent newly developed tumor foci. At histopathological analysis of hepatectomy, the presence of satellite nodules and portal vein invasion herald tumor metastases and early recurrence. The transplantation team should not 
rush to salvage LDLT except after considering the biological aggressiveness of the recurrent tumor and the likelihood of re-recurrence into the implanted neo-liver [22].

\subsection{Sequential LDLT and preemptive LDLT}

They are new categories with no consensus, performed in some centers within institutional protocols. In preemptive LDLT, cases that underwent initial hepatectomy and showed adverse histopathological criteria like microvascular invasion or satellite tumor nodules are prepared for LDLT without unnecessary waiting for the inevitable expected recurrence [21, 23, 24].

In sequential LDLT, pretransplant loco-regional therapy (LRT) is administered aiming at complete pathological necrosis of the index tumor before LDLT for cases within accepted criteria. Moreover, LDLT is performed after a scheduled short interval due to donor availability. The exact duration of the interval is not yet determined. Some centers perform LDLT after 3 months from the time of pretransplant therapy. This concept developed from the cumulative experience of histopathological assessment of explanted livers and the impact of pathological necrosis on post LT outcomes. HCC patients who achieve pathological necrosis $\geq 60 \%$ have significantly better overall survival and recurrence-free survival [25].

The employment of salvage LDLT is sometimes controversial. Some transplant surgeons, in view of certain radiological, pathological and biological hallmarks, don't prefer to wait for the inevitable recurrence or deterioration of patient or liver conditions to occur (preemptive LDLT). Some argue that the readily available donor associated with vigilant surveillance can always tailor the transplantation in the right time for the best outcome (salvage LDLT).

\section{Evolution of selection criteria}

Over the previous two decades, criteria of LT had been expanded and modified from morphological and nonmorphological perspectives. Nevertheless, Milan criteria (MC) keep their place as the gold standard for LT. The long-term outcome and prognostic impact of LT within MC had been reproducibly studied with consistent excellent results [19, 26].

\subsection{Why the criteria for LDLT had been expanded?}

The MC advocated by Mazzaferro et al. [27] in 1996 are considered the base upon which several centers around the world validated their experiences in liver transplantation. These first clearcut criteria were published in the New England journal of medicine as a practice changing and ground breaking progress in HCC treatment. That is because MC provided posttransplant survival rates of $75-95 \%$ at 2 years and $70-80 \%$ at 5 years in several studies. Indeed, a metaanalysis of published data has confirmed the survival advantage of MC and its association with a low risk of selecting patients with aggressive tumors [19, 27-29].

However, Milan criteria have been expanded and modified steadily for the following reasons: 
1. They were considered too restrictive and many reports showed similar outcomes for cases beyond Milan [13, 30].

2. The growing evidence of the clear contribution of nonmorphological tumor characteristics to the outcome of patients after transplantation. So, the static "morphological" Milan criteria were expanded and modified. The other pathological and biological tumor criteria include: micro-vascular invasion, alpha-feto protein (AFP) level, deoxy-gamma carboxy prothrombin (dGCP) level and tumor avidity on PET scan [31-35].

3. The observation of two incorrectly estimated groups: an underestimated group that had been transplanted within MC but showed worse outcome and an overestimated group that was beyond MC and had comparable outcome after LDLT $[35,36]$.

\subsection{Selection criteria according to different centers of excellence (Table 1) [27, 37-45]}

\begin{tabular}{|c|c|c|c|}
\hline \multicolumn{4}{|c|}{ Selection criteria of liver transplantation in different series and centers of excellence } \\
\hline Criteria & Parameters & Type of parameters & Over-all survival \\
\hline Milan & $\begin{array}{l}\text { Solitary tumor } \leq 5 \mathrm{~cm}[\mathrm{~T} 1] \text {, up to } 3 \text { tumors non }>3 \mathrm{~cm} \text {, no } \\
\text { macrovascular invasion }\end{array}$ & Morphological only & $85 \%$ (4-year) \\
\hline UCSF & $\begin{array}{l}\text { Solitary HCC } \leq 6.5 \mathrm{~cm} \text {, or } \leq 3 \text { nodules with the largest } \\
\text { lesion } \leq 4.5 \mathrm{~cm} \text { and a total tumor diameter } \leq 8 \mathrm{~cm}\end{array}$ & Morphological only & $75 \%$ (5-year) \\
\hline $\begin{array}{l}\text { Modified Milan } \\
\text { (Up to } 7)\end{array}$ & Tumor number + size of the largest tumor $(\mathrm{cm}) \leq 7$ & Morphological only & $71 \%$ (5-year) \\
\hline Toronto & $\begin{array}{l}\text { Any tumor size or number, if pretransplant tumor biopsy } \\
\text { showed no poor differentiation, no major vascular } \\
\text { invasion, no extrahepatic disease }\end{array}$ & $\begin{array}{l}\text { Morphological- } \\
\text { pathological }\end{array}$ & $72 \%$ (5-year) \\
\hline Kyoto University & $\begin{array}{l}\leq 10 \text { tumors, all } \leq 5 \mathrm{~cm} \text { and the serum level of des. Gama } \\
\text { carboxy prothrombin }(\mathrm{d} . \mathrm{GCP}) \leq 400 \mathrm{mIU} / \mathrm{ml}\end{array}$ & $\begin{array}{l}\text { Morphological- } \\
\text { biological }\end{array}$ & $87 \%$ (5-year) \\
\hline $\begin{array}{l}\text { Kyushu } \\
\text { University }\end{array}$ & $\begin{array}{l}\mathrm{HCC} \leq 5 \mathrm{~cm} \text { and a serum d.GCP } \leq 300 \mathrm{mIU} / \mathrm{ml} \text {. It had } \\
\text { been refined by adding neutrophil to lymphocyte ratio } \\
(\mathrm{NLR})>4\end{array}$ & $\begin{array}{l}\text { Morphological- } \\
\text { biological }\end{array}$ & $83 \%$ (5-year) \\
\hline Tokyo University & $\begin{array}{l}5-5 \text { rule: } \mathrm{HCC} \text { not larger than } 5 \mathrm{~cm} \text { and no more than } 5 \\
\text { nodules }\end{array}$ & Morphological only & $75 \%$ (5-year) \\
\hline $\begin{array}{l}\text { Asan Medical } \\
\text { Center }\end{array}$ & $\begin{array}{l}\text { HCC not larger than } 5 \mathrm{~cm}, 6 \text { or fewer nodules without } \\
\text { gross vascular invasion }\end{array}$ & Morphological only & $82 \%$ (5-year) \\
\hline Hangzhou & $\begin{array}{l}\mathrm{HCC} \geq 8 \mathrm{~cm} \text { if serum alpha-fetoprotein level } \leq 400 \mathrm{ng} / \mathrm{ml} \\
\text { and only grade I or II }\end{array}$ & $\begin{array}{l}\text { Morphological, } \\
\text { biological, and } \\
\text { pathological }\end{array}$ & 72.\% (5-year) \\
\hline
\end{tabular}

Table 1. Different selection criteria for liver transplantation adopted in most of transplantation in the world. Note that the main western criteria (Milan, UCSF, UP to 7) depend largely on morphology. Eastern-based criteria (KyotoKyushu-Hangzhou) had modified by incorporating biological behavior of the tumors. The Toronto criteria are unique in its sole dependence on pretransplant biopsy. 
Milan criteria were based on preoperative imaging and validated in many institutions all over the world [27]. In 2001, Yao et al. in the University of California San Francisco (UCSF) expanded the morphological criteria based on pathological assessment of explanted livers and then they prospectively validate their criteria based on pretransplant imaging in 2007 [37, 46]. Though this set of criteria is applied successfully in many regions, it has not been applied on a wide scale because they allow transplantation for large HCCs $(6.5 \mathrm{~cm}$, representing a tumor volume of $144 \mathrm{~cm}^{3}$ ), which had been associated with worse outcomes in some studies. UCSF criteria (like Milan criteria) also exclude all patients with more than three lesions, some of whom may have the same outcome as those within criteria [47]. The 3- and 5-year survival rates after LDLT based on the UCSF criteria range between $78-96 \%$ and $66-90 \%$, respectively, in different centers [28]. This wide variation of survival outcomes may also be attributed to variations of the surgical outcome and early mortality.

It should be emphasized that there are no consensus criteria for LDLT allocation. Each individual institution adopts one of the current criteria, tailoring treatment to their unique patient and general circumstances. Most of centers with high experience in LDLT for HCC currently focus on modification rather than numerical expansion of the number and size of tumors. The incorporation of biological factors (tumor markers, inflammatory markers) or available pathological factors from previous hepatectomy help select best candidates and hence predict the outcome and protect against recurrence $[29,35,48]$.

\section{Preoperative evaluation}

Liver transplantation under optimal circumstances is a clear benefit of LDLT. The procedure can be carried out in a timely fashion after exhaustive assessment of tumor burden by different imaging modalities and biological markers. The tumor burden can then be manipulated by pretransplant therapies using the different loco-regional treatment options. Preoperative evaluation of the donor is not much different from the nonmalignant cases of LDLT except for some additional ethical perspectives as discussed previously. For the recipient, the details of preoperative assessment are beyond the scope of this chapter. However, we consider some important points in the pretransplant setting.

\subsection{Imaging}

Preoperative imaging of the recipient aims at assessment of tumor burden and suitable metastatic workup, for occult HCC or other cancer types. Dynamic CT or MRI with arterial enhancement is the standard of care according to high level of evidence. Extrahepatic staging should include CT chest and CT or MRI of abdomen and pelvis [12, 23].

The discrepancy between preoperative imaging and real tumor burden in explanted livers had been investigated in many centers. The state-of-the-art imaging modalities are not $100 \%$ accurate. CTA and MRI were both accurate to determine whether patients fit within the Milan or UCSF criteria, but CTA was slightly better than MRI to evaluate tumor number and size [49]. In the current era of pretransplant invasive therapies, the liver background became more 
confusing due to the presence of regenerative nodules, necrotized tumors, and other small tumors [35].

The incidental finding of pulmonary nodule(s) on pretransplant CT warrants appropriate evaluation. Many studies had determined a cutoff point to the size of pulmonary lesion at $5 \mathrm{~mm}$. A lesion $<5 \mathrm{~mm}$ is less likely to be malignant and if remained stable or showed regression over a period of 3-6 months, liver transplantation is performed. For a lesion $>5$ $\mathrm{mm}$, a biopsy is needed to rule out malignant nature that contraindicates transplantation. If this lesion was the same in repeated images over previous 2 years, the possibility of infection is most likely and management is done accordingly. Video-assisted thoracic surgery (VATS) is perfectly employed for an excision biopsy of these small pulmonary nodules [50].

\subsection{Alpha-feto protein (AFP)}

This biological tumor marker plays important role in the assessment of tumor burden and aggressiveness although many other markers have been used in clinical practice over the past two decades. The AFP value has its clinical significance either in the preoperative or the posttransplant settings. In addition, the dynamic changes to its value in relation to downstaging therapy had been explored. An important question in the preoperative setting is its role as an exclusion tool, either alone or in combination of other radiological, biological, or pathological markers.

Some studies check the cutoff level above which patients should not undergo LT. At an AFP level $>1000 \mathrm{ng} / \mathrm{ml}$, approximately $5 \%$ of patients would be excluded from LT with $20 \%$ reduction in the rate of HCC recurrence. If a lower cut-off level is applied, this would result in a greater reduction in the recurrence rate but at the expense of excluding many more patients from LT who might not have tumor recurrence and would have benefited from LT. Also, patients who had decline of an initial AFP level $>1000 \mathrm{ng} / \mathrm{ml}$ after loco-regional therapy had a more favorable prognosis after LT. Alpha feto-protein level $<1000$ has thus been combined to the UCSF criteria for patient selection in some centers [51].

More recently, a prospective multicenter study has explored the use of a combination of total tumor volume (TTV) $\leq 115 \mathrm{~cm}^{3}$ and AFP $\leq 400 \mathrm{ng} / \mathrm{ml}$ as an exclusion tool from LT in centers with a median waiting time of 8 months. This study took an advantage from a previous observation of the inferior survival outcome of patients who are within MC but have the AFP level $\geq 400 \mathrm{ng} / \mathrm{ml}$. In this study, patients who were beyond MC but within TTV/AFP had survival outcome similar to cases within MC [29].

Many other biomarkers can be evaluated in the pretransplant setting to predict the posttransplant outcome. A recent study in our center showed that repeated measurement of serum levels of novel biomarkers including fibroblast growth factor-2 (FGF-2), survivin, Ki67, endostatin, and vascular endothelial growth factor (VEGF), before and after LDLT for HCC, could predict HCC recurrence [52]. 


\subsection{Fludro-deoxy glucose-positron emission tomography (FDG-PET) scan}

Pretransplant 18F-FDG uptake on PET scan in liver transplant candidates with HCC is a useful additional parameter for the evaluation of tumor biology. Positive preoperative PET scans are significantly associated with an increased risk of posttransplant HCC recurrence and inferior outcome, an observation that relates a positive PET status with the presence of microvascular invasion in explant tumor pathology [31]. In a recent Korean study, the combination of the positive FDG-PET study and AFP level $>200 \mathrm{ng} / \mathrm{ml}$ could predict tumor recurrence more precisely after LDLT than Milan criteria [35]. More recently, a retrospective study at our center has showed that the combination of UCSF criteria with the FDG-PET status can predict tumor recurrence after LDLT and that tumor recurrence is earlier in cases with positive FDG-PET results. On the basis of the maximal standardized uptake value (SUVmax), high-risk group cases (positive study with $S U V_{\max } \geq 5$ ) had worse recurrence-free survival compared with lowrisk cases (positive study with $\mathrm{SUV}_{\max }<5$ ) [53].

\subsection{Donor characteristics}

In addition to the previously discussed controversy of impact of donor type (living or deceased) on HCC recurrence, other donor factors have been studied. A recent review of the American Scientific Registry of Transplant Recipients (SRTR) that involved 9724 patients revealed that the following donor factors were independently associated with high risk of HCC recurrence after transplantation: high BMI, history of DM, and severe graft steatosis. All the previous factors are evident at the time of organ allocation and must be considered before transplantation [54].

\section{Loco-regional therapy (LRT)}

The pretransplant therapies are implemented heavily for HCC, being an essential component of HCC treatment algorithms in most of treatment guidelines. Earlier in the treatment of HCC roadmaps, they were considered "bridging" tools to keep the patient chances of LT during the waiting time. Currently, a realized value of LRT is their ability to probe the tumor's internal milieu, acting as a selecting tool based on the degree of tumor's response as an indicator of its aggressiveness. So, adopting such therapies gives the time to observe the tumor's behavior before the ultramajor costly step of liver transplantation [55]. Bypassing such an observation period in the setting of multifocal or locally advanced tumor risks transplanting patients with systemic disease.

\subsection{HCC downstaging before liver transplantation}

The difference between downstaging and neoadjuvant therapies is that in the former the tumor status is beyond the proposed criteria for transplantation and LRT is administered to reduce the tumor burden and to render the case fit for transplantation. In neoadjuvant therapy, the patient is already within criteria but treatment is given to prevent tumor progression to the 
"beyond criteria" state while waiting for the donor and to induce tumor necrosis aiming at better long-term outcome [56].

In different series from Asian centers, more than $30 \%$ of patients who were beyond Milan/ UCSF criteria were effectively downstaged to the "within criteria" state with a 5-year survival rate similar to the initially fit cases $[57,58]$. A meta-analysis explored the effect of downstaging to Milan criteria, and the success rate exceeded $40 \%$. However, the recurrence rate was as high as $16 \%$ [59].

TACE and RFA are the main tools of downstaging. TACE mediates its effect by inducing complete pathological necrosis with response rates ranging between 27 and $57 \%$. RFA is used For accessible lesions to induce coagulative necrosis, either alone or in combination with liver resection [60]. In LDLT, timing of transplantation after a period of waiting from the time of LDLT is possible. Most centers wait for a period of 3-6 months to evaluate tumor progression as an indicator of aggressiveness before embarking into LDLT.

Though widely applicable in most HCC guidelines, there are no unified criteria either for selecting cases for downstaging or for determining them as good responders and hence listing them for LT. This fact highlights the wide variation of transplantation criteria among different regions and centers. As a rule of thumb, the criteria to enter a downstaging program should include patients who have well-defined and acceptable chances of good outcomes after transplantation if the downstaging goal is achieved [56]. In our center, not only we apply downstaging for cases beyond UCSF criteria, but also we consider cases with high AFP or high FDG uptake on PET scan for downstaging. In our early experience, patients were downstaged to fit Milan criteria. Our initial results of 35 patients were encouraging with a 5-year survival rate of $90 \%$ and those patients are still disease-free up to date, after follow-up of 10 years. Since July 2006, our criteria were extended to UCSF criteria. In our experience of 161 HCC patients, $51(31.6 \%)$ were successfully downstaged to fit the UCSF criteria. The overall 1- and 5-year survival rates for downstaged cases were 94.1 and $92.7 \%$, respectively. The recurrence rate was $9.2 \%$. The survival rates were similar for different pretransplantation downstaging procedures [57].

\section{Operative/surgical highlights}

The first successful case of liver transplantation in Asia was performed by Chen in 1984 in Chang Gung Memorial Hospital, Taiwan. From the cumulative experience at our center, we would like to emphasize that a sound surgical procedure is mandatory for the best outcome. It has been postulated that the experience of the transplant team is an important factor that independently affects the outcome though the learning curve of liver transplant can be steep and painful in some centers launching an LDLT program. In essence, we prefer not to correct recipient coagulopathy before surgery, because many studies showed no value of preoperative correction. Donor hepatectomy should be accomplished with minimal blood loss, obviating the need for blood transfusion. The routine use of microsurgical procedure is performed in our center for arterial anastomosis as well as biliary reconstruction. Surgical outcome largely 
affects survival and hence final outcome. Early postoperative vascular complications are the main reasons of graft loss and in-hospital mortality and must be managed vigorously [49, 6164].

\subsection{Timing of donor and recipient surgeries}

In nonmalignant cases of LDLT, the recipient surgery is usually begun after the donor surgery commenced till confirmation of feasible biliary anatomy by cholangiography. In the early experience of LDLT for HCC, some surgeons preferred to confirm resectability of the liver containing cancer and the absence of lymph node metastases through a smaller incision before proceeding with graft harvesting from the donor [65, 66]. However, the current imaging modalities surpass this step and allow precise decision in advance. Most experienced centers largely depend on high quality CT or MRI images and the timing is adopted like nonmalignant cases of end-stage liver failure.

\subsection{Safety margin}

A proper safety margin is an integral part of the sound oncological surgical procedure. Some peripheral lesions may be found in very close proximity to or even adherent to parietal peritoneum, diaphragm, omentum, or less commonly small or large bowl loops. A previous hepatectomy increases the adhesions with the aforementioned structures. It is thus convenient to take a proper safety margin through excision of parts of peritoneum and diaphragmatic muscle. Very infrequently, small or large bowl resection and anastomosis may be required. Reexploration for additional safety margin after final pathological assessment is a very hazardous procedure for such a frail patient, but may be warranted in some conditions according to institutional experience.

\subsection{Management of nodal disease}

In a recent review from the International Registry of Hepatic Tumors in Liver Transplantation, the incidence of lymph node metastases in cirrhotic patients with HCC undergoing LT was $6.5 \%$. Hilar lymph node (LN) involvement in cases of HCC constitutes a contraindication for liver transplantation because of dismal prognosis. Recent imaging techniques are able to identify enlarged suspicious lymph nodes in the pretransplant setting. However, the frequent presence of LN enlargement due to chronic inflammation in cases of hepatitis C-induced cirrhosis may make the diagnosis less clear. It is convenient to perform frozen section evaluation for suspicious nodes unexpectedly encountered during recipient operation. The decision to proceed or abort the procedure in the case of inconclusive frozen section analysis or the unavailability of frozen section depends largely on the experience of the transplant team. According to a meta-analysis study, systematic hilar lymphadenectomy during LT for HCC should routinely be undertaken, especially in the context of coexisting hepatitis C or secondary biliary cirrhosis. Some centers recommend harvesting of at least four lymph nodes at the time of liver transplantation but the debate remains open with no available consensus on the extent of such lymphadenectomy $[67,68]$. 


\subsection{The middle hepatic vein (MHV)}

The MHV is a controversial topic within the LDLT procedure in general. In the case of LDLT for HCC, this issue should be taken more seriously. It has been postulated that the resultant venous congestion from improper management of tributaries of MHV is a possible factor favoring tumor recurrence. Congestion of the graft leads to impediment of portal inflow with subsequent ischemia in the congested region and compensatory accelerated regeneration of the well-drained areas of the graft. Consequently, the cellular changes in response to both processes may enhance tumor recurrence [69].

\subsection{IVC management (see Figure 1)}

The observation of HCC recurrence after LDLT in the hepatic vena-caval wall has shed light on the possibility of microvascular infiltration with consequent local and distant recurrence. This finding triggered the concept of aggressive en-bloc caval resection and reconstruction during LDLT. This step is also justified by another two facts: first, it prevents a positive margin of latent cancer in caval branches; second, the least amount of manipulation of the native liver with HCC should be employed to avoid and control distant metastasis after LDLT [70].

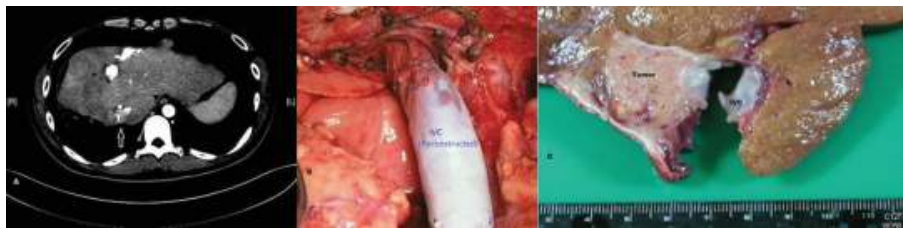

Figure 1. A case of HCC subjected to LDLT. The tumor (T) was intimately adherent to the IVC wall without apparent invasion in pretransplant CT angiography (A; arrow). During the recipient operation, the liver was explanted en-bloc with the IVC segment adjacent to the tumor. Reconstruction of the IVC was carried out using cryo-preserved iliac v. graft (B). Final gross pathology shows no intraluminal transgression by the tumor (C).

\subsection{Complications of LDLT of the manipulated liver, i.e., after loco-regional therapy}

Extensive adhesions are commonly encountered following previous liver resection in case of salvage liver transplantation. Tough vascular adhesions usually form between a large hepatectomy site and small intestine and omentum. Sharp dissection and meticulous hemostatic control would reduce the difficulty of subsequent transplantation and decrease the incidence of postoperative complications [71]. Laparoscopic liver resection is now performed on a wide scale in many centers. One of the most prominent advantages of laparoscopic initial hepatectomy is the marked reduction of adhesions as evidenced in subsequent liver transplantation in many series thus facilitating the procedure [72].

Intimal dissection (ID) of the recipient hepatic artery has emerged as a grave complication that may occur after TAE or TACE. Intimal dissection if not discovered and managed properly may end up in graft loss. Gentle handling of HA is mandatory during LT to prevent aggravation or precipitation of dissection in the fragile HA. Once this complication is suspected intraopera- 
tively, microscopic assessment is needed for proper decision. Either trimming and discarding the dissected segment or discarding the whole native HA and reconstruction may be considered, hence the importance of microvascular expertise in the LDLT team [73].

\section{Ethical considerations}

Two main ethical pillars of the decision of LDLT for management of HCC must be considered. First, donor safety and optimizing his or her care is no different in LDLT for HCC compared to that for nonmalignant cases of liver failure. Second, the procedure must provide an acceptable outcome to the recipient compared to DDLT. From the ethical point of view, LDLT is justified, as long as the proposed regional criteria are strictly followed, being MC or any scientifically justified institutional expanded or modified criteria. Noteworthy that there is no consensus about what risk to the living donor might be considered acceptable for a given risk of recurrence $[13,74]$.

With increased awareness and patient education facilities, the ethical responsibilities regarding patient counseling became more complex. It is not all about the perioperative complications. Recently, ethical considerations took a very different perspective, that is offering LDLT to highrisk patients and excluding others who are low-risk patients based on imaging criteria only. As mentioned earlier, two wrongly estimated groups are revealed: cases within criteria that have aggressive biological behavior and cases beyond criteria with indolent tumors. Two ethical situations subsequently ensued; the first is the risk of recurrence of biologically aggressive tumors even when strict criteria are adhered to, i.e., the underestimated group. The second is about the loss of chance of LT in the other group. The transplantation team should never rush to transplantation in response to family pressure and readily available donor without proper discussion of all that issues [35].

When the partial graft fails for any reason, a special-but very uncommon-ethical situation is encountered because normally it requires an urgent deceased donor retransplantation. It has been established that if LDLT was used in a situation where a deceased donor is contraindicated, such as exceeding the accepted criteria for deceased donation, the patient should not be retransplanted because he/she would not be transplanted initially if on wait-list for DDLT [12, 66]. Finding another living donor must be balanced against the expected outcome of the procedure. There are no clear-cut decisions to such situations and the institutional experience would tailor the right decision.

When talking about salvage LDLT, the patient may be reluctant to undergo the procedure even with the availability of a donor. Some patients may argue that they want to be alive more but do not want to harm anyone. In these circumstances, the transplant team should alert the patient about how long can he/she delay the procedure without a considerable risk of tumor progression or being transferred into the nontransplantable state. A study from Japan revealed that patients who receive downstaging therapy can wait no more than 12 months. However, the exact duration is not determined and the management plan should be tailored to each case [75]. 


\section{Controversial topics}

The increasing number of HCC cases, the parallel progress in expertise of LDLT, and the dynamic changes in the selection criteria all enriched the liver transplantation community with many important topics and debates. A lot of studies were carried out to find answers or to reach a consensus in many of these topics. However, the retrospective nature of most studies, the different reporting methodologies and small sample sizes are all flaws that hampered the appearance of high level of evidence to address all topics.

\subsection{What is the best treatment option of early HCC? Resection or liver transplantation? Primary or salvage approach?}

- The surgical decision can be either LR or LT in 20-25\% of cases of HCC amenable for surgery. Liver transplantation is hypothetically superior to resection because it removes the cancer and its underlying pathology. For many patients it is not possible to perform resection because of the tumor size, anatomical location of the tumor, or poor liver function, and liver transplantation is the only surgical option [76]. However, even in many Asian centers which perform LDLT on regular basis, up to $50 \%$ of early HCC cases may not have suitable donors or be concerned about the risk to donor. So, many Asian centers adopt the policy of offering LR to patients with resectable tumors with compensated cirrhosis and deferring LT to the salvage setting [13, 23, 77, 78].

- The controversy regarding LR or LT in HCC patients is largely confined to early cases with a well-preserved liver function. There are no randomized controlled trials that addressed this issue through a head-to-head comparison. Given that organ shortage for LT is a persistent challenge worldwide and also the presence of underserved areas with deficient LT programs, the identification of cases that would obtain similar survival outcomes when either submitted to LR or to LT is of paramount importance [79, 80].

- The strategy of offering LR for simultaneously resectable and transplantable cases was based on the observation from many studies that $20-30 \%$ of HCC cases that had LR may not witness recurrence for $>5$ years. In addition, with vigilant surveillance programs, most of recurrent cases would fit into the $\mathrm{MC}$ and can be transplanted under optimal conditions due to the availability of the living donor, with no negative impact on the outcome. This obviates many patients the downside of early immunosuppression [5]. In addition, the mortality rate of LR at an experienced center is less than $2-5 \%$, and overall survival rates were comparable to primary LT $[4,24]$.

- The Barcelona Clinic Liver Cancer approach: Resection is the treatment of choice in patients with very early stage HCC and normal bilirubin levels as well as the absence of portal hypertension. If the liver functions are impaired, LT is recommended as a primary treatment approach. In the early stage of HCC, it has been found that the 10-year outcome after LT is superior to LR in view of the latter's well-known higher recurrence risk. So, the choice of primary LT is recommended. The same approach is adopted by the American Association of Study of Liver Disease (AASLD) guidelines that prefer liver resection for very early cases with the optimal liver profile [7, 78, 81, 82]. 
- A meta-analysis of studies that involved patients within MC revealed a survival advantage for LT over LR [83]. In a more recent meta-analysis that involved 1572 patients in whom both LR and LT are feasible, the authors did not find any survival advantage of LT compared with LR. They concluded-based on low quality evidence-that LR is preferable to LT in patients who are feasible for both options [84].

- Conclusion: the overall survival advantage of LT is unclear, though recurrence-free survival is definitely better with LT. The main drawback for LT-especially LDLT - is the relatively increased mortality in the early postoperative period. In high volume centers where LDLT is performed at high rates, perioperative mortality is reduced significantly. So, a clear overall survival advantage for LT may be evident [85]. For a definite evidence-based conclusion, a randomized controlled trial comparing LT and LR is needed, a condition that is very difficult due to practical and ethical restrictions. Proper patient selection and optimization of criteria according to the pretransplant setting (primary or secondary) would help make a treatment roadmap.

\subsection{Living donor or deceased donor for HCC?}

One of the most critical controversies in the transplantation field is the possibility of an increased rate of recurrence of HCC after LDLT compared to DDLT.

Several hypotheses have been postulated to figure out this possibility:

- The fast tract effect: due to the shortened waiting time for LDLT, progression of HCC with aggressive tumor biology might not be recognized during such a short-waiting time. This is in contrary to the situation in DDLT, where the long-waiting time can naturally select cases with indolent behavior.

- The growth factors and cytokines released during rapid regeneration of the partial grafts might contribute to tumor progression and recurrence.

- The extensive dissection and mobilization of the liver might increase the feasibility of tumor dissemination through the hepatic vein and increased potential for leaving residual tumor cells.

- The exaggerated vascular inflow associated with small size grafts elicits vast angiogenesis with subsequent carcinogenic effect.

- The less radical hepatectomy in LDLT with the native IVC left in place may constitute a suboptimal oncologic procedure $[15,55,86-88]$.

A multi-center study was carried out on the database of China Liver Transplant Registry (CLTR) to highlight this issue. They explored the data of 6860 patients (6471 (DDLT) and 389 LDLT and concluded that both overall survival and recurrence-free survival were comparable between both approaches [89]. In an international consensus conference of liver transplantation (Zurich 2012), it had been stated that no convincing difference in outcome could be identified according to the type of graft, although a higher risk of recurrence was noted in fasttracked patients. The experts advised an interval of observation for the biological behavior of 
the tumor to manifest. A period of 3 months had been suggested [12]. Many other groups reported similar recurrence rates after LDLT and DDLT and also similar long-term outcomes $[14,87]$.

However, others have found significantly higher rates of tumor recurrence after LDLT compared with DDLT for HCC. The Adult to Adult Living Donor Liver transplantation (A2ALL) study reported a significantly higher 3-year tumor recurrence rate after LDLT [15, $88,90,91]$. One should consider that many studies that reported better outcome of DDLT than LDLT are conducted in the west and some of them were flawed by small sample sizes and the possibility of selection bias since DDLT was applied mostly on cases within Milan criteria only and those beyond Milan criteria are relegated to LDLT [92].

A systematic review and meta-analysis addressed this question (Is the regenerating liver of LDLT a fertile environment for HCC recurrence?). Though no randomized controlled trials were included, they came to a similar conclusion; disease-free survival (DFS) is worse after LDLT compared with DDLT but there was no difference observed in overall survival (OS). In the same meta-analysis, there was a concern that the follow-up periods in the included studies are probably too brief to detect a major impact on survival resulting from differences in disease recurrence. The same study revealed that the overall survival after LDLT vs. DDLT was better -though not statistically significant-in studies from eastern centers compared with studies from western centers. This may reflect differences between these regions in patient selection or case-volumes effects on surgical outcomes [6]. In conclusion, to date there is no definite evidence supporting higher recurrence after LDLT than DDLT $[55,92]$.

\subsection{Pre-LDLT liver biopsy, can the trend be changed?}

Because the risk of tumor seeding may be $2-4 \%$ along the needle biopsy tract and thus increasing the risk of metastases, liver biopsy should be considered only when there is uncertainty as to the diagnosis. This might occur, for example, in the presence of an HCC arising in the noncirrhotic liver or contradictory clinical, laboratory, and radiological findings [81]. However, a biopsy has the potential problems of sampling error. For example, different nodules may have different grades within the index tumor. It has also been demonstrated that even in a single-needle biopsy, adjacent tumor cells can be of different degrees of differentiation. Nevertheless, if transplantation is planned for HCC beyond the standard criteria, tumor biopsy appears to be a logical approach if there is suspicion of diagnosis [28].

Dubay et al. [44] have provided their experience in the University of Toronto based solely on a liver biopsy before LT. They have no size or number restriction for LT. They concluded that a protocol using a biopsy to exclude poorly differentiated tumors achieved excellent survival rates $(70 \%$ at 5 years) [44].

\subsection{Can we exclude morphological selection criteria in the coming era?}

Morphological criteria of HCC describe the appearance, while biological criteria describe the concealed behavior. Ultimately, the behavior of HCC is the final-deciding factor on patient outcome. As shown earlier, modification of morphological criteria is pursued in many studies 
with the incorporation of biological factors [13]. In a study from Korea, the recipient selection based on pure biological factors was proposed. The group showed that FDG PET positivity with a cutoff point of maximum systemic uptake value $\left(\mathrm{SUV}_{\max }\right)$ of 1.1 combined with the AFP serum level at $400 \mathrm{ng} / \mathrm{ml}$ is able-alone-to predict patient outcome more precise than morphological criteria [35]. More studies are needed to explore nonmorphological selection processes.

\section{Conclusions}

LDLT is a robust treatment pathway for HCC. Indeed, it may be the only pathway in this era of organ shortage especially in some Asian countries. The employment and dynamic application of selection criteria of LDLT for HCC are the cornerstone to acquire the best outcome. The number and size of the tumor(s) are not the only factors to consider for patient selection but some other factors are also present. Most of transplantation centers are combining biological (AFP-tumor avidity in PET scan) and pathological (pretransplant biopsy) factors of the tumors to the morphological factors aiming at refined patient selection for the optimal outcome. In the current era of personalized medicine, treatment of HCC should be tailored according to each individual patient and tumor criteria. A sound oncological surgical procedure is the core of successful LDLT and has its direct impact on the final outcome.

\section{Author details}

Chih-Che Lin $^{1 *}$, Ahmed Mohammed Abdel Aziz Elsarawy ${ }^{2}$ and Chao-Long Chen ${ }^{1}$

*Address all correspondence to: immunologylin@gmail.com

1 Department of general surgery and liver transplant center, Chang Gung Memorial Hospital, Kaohsiung, Taiwan

2 Liver transplant center, Chang Gung Memorial Hospital, Kaohsiung, Taiwan

\section{References}

[1] Knox JJ, Cleary SP, Dawson LA. Localized and systemic approaches to treating hepatocellular carcinoma. J Clin Oncol. 2015;33(16):1835-44. Available from: http:// jco.ascopubs.org/content/33/16/1835.long

[2] Bruix J, Gores GJ, Mazzaferro V. Hepatocellular carcinoma: clinical frontiers and perspectives. Gut. 2014;844-55. 
[3] Sonnenday CJ. Recurrent hepatocellular carcinoma after living donor liver transplantation: a preventable problem or an acceptable risk? Ann Surg Oncol. United States; 2010;17:2262-3.

[4] de Villa VH, Lo C-M, Chen C-L. Ethics and rationale of living-donor liver transplantation in Asia. Transplant. 2003;75(3 Suppl):S2-5. Available from: http://www.ncbi.nlm. nih.gov/pubmed/12589129

[5] Chen C-L, Concejero AM. Liver transplantation for hepatocellular carcinoma in the world: the Taiwan experience. J Hepatobil Pancreat Sci. Japan. 2010;17(5):555-8.

[6] Grant RC, Sandhu L, Dixon PR, Greig PD, Grant DR, McGilvray ID. Living vs. deceased donor liver transplantation for hepatocellular carcinoma: a systematic review and meta-analysis. Clin Transplant. Denmark; 2013;27(1):140-7.

[7] Dufour JF, Greten TF, Raymond E, Roskams T, De T, Ducreux M, et al. EASL-EORTC clinical practice guidelines: management of hepatocellular carcinoma. J Hepatol. 2012;56(4):908-43. Available from: http://linkinghub.elsevier.com/retrieve/pii/ S0168827811008737

[8] Torre LA, Bray F, Siegel RL, Ferlay J. Global cancer statistics. CA Cancer J Clin. 2015;65(2):87-108.

[9] Shirabe K, Taketomi A, Morita K, Soejima Y, Uchiyama H, Kayashima H, et al. Comparative evaluation of expanded criteria for patients with hepatocellular carcinoma beyond the Milan criteria undergoing living-related donor liver transplantation. Clin Transplant. Denmark; 2011;25(5):E491-8.

[10] Chen C-L, Kabiling CS, Concejero AM. Why does living donor liver transplantation flourish in Asia? Nat Rev Gastroenterol Hepatol. 2013;10(12):746-51. Available from: http://www.ncbi.nlm.nih.gov/pubmed/24100300

[11] Khalaf H, Marwan I, Al-Sebayel M, El-Meteini M, Hosny A, Abdel-Wahab M, et al. Status of liver transplantation in the Arab world. Transplantation. 2014;97(7):722-4. Available from: http://www.ncbi.nlm.nih.gov/pubmed/24603475

[12] Clavien P-A, Lesurtel M, Bossuyt PMM, Gores GJ, Langer B, Perrier A. Recommendations for liver transplantation for hepatocellular carcinoma: an international consensus conference report. Lancet Oncol. England; 2012;13(1):e11-22.

[13] Mazzaferro V, Chun YS, Poon RTP, Schwartz ME, Yao FY, Marsh JW, et al. Liver transplantation for hepatocellular carcinoma. Ann Surg Oncol. United States; 2008;15(4):1001-7.

[14] Bhangui P, Vibert E, Majno P, Salloum C, Andreani P, Zocrato J, et al. Intention-to-treat analysis of liver transplantation for hepatocellular carcinoma: living versus deceased donor transplantation. Hepatology. United States; 2011;53(5):1570-9. 
[15] Ng KK, Lo CM. Liver transplantation in Asia: past, present and future. Ann Acad Med Singapore. Singapore; 2009;38(4):310-22.

[16] Chen C, Cheng Y, Yu C, Ou H, Tsang LL, Huang T, et al. Living donor liver transplantation: the Asian perspective. Transplantat. 2014;97 Suppl 8: S3.

[17] Barr ML, Belghiti J, Villamil FG, Pomfret EA, Sutherland DS, Gruessner RW, et al. A report of the vancouver forum on the care of the live organ donor: lung, liver, pancreas, and intestine data and medical guidelines. Transplantat. 2006;81(10):1373-85. Available from: http://content.wkhealth.com/linkback/openurl?sid=WKPTLP:landingpage\&an= 00007890-200605270-00004

[18] Nadalin S, Bockhorn M, Malagó M, Valentin-Gamazo C, Frilling A, Broelsch CE. Living donor liver transplantation. HPB (Oxford). 2006;8(1):10-21. Available from: http:// www.pubmedcentral.nih.gov/articlerender.fcgi?artid=2131378\&tool=pmcentrez\&rendertype $=$ abstract

[19] Mazzaferro V, Bhoori S, Sposito C, Bongini M, Langer M, Miceli R, et al. Milan criteria in liver transplantation for hepatocellular carcinoma: an evidence-based analysis of 15 years of experience. Liver Transpl. United States; 2011;17 Suppl 2:S44-57.

[20] Farkas S, Hackl C, Schlitt HJ. Overview of the indications and contraindications for liver transplantation. Cold Spring Harb Perspect Med. United States; 2014;4(5).

[21] Majno PE, Sarasin FP, Mentha G, Hadengue A. Primary liver resection and salvage transplantation or primary liver transplantation in patients with single, small hepatocellular carcinoma and preserved liver function: an outcome-oriented decision analysis. Hepatology. United States; 2000;31(4):899-906.

[22] Lee HS, Choi GH, Joo DJ, Kim MS, Choi JS. The clinical behavior of transplantable recurrent hepatocellular carcinoma after curative resection: implications for salvage liver. Transplantation. 2014;2717-24.

[23] Concejero A, Chen C-L, Wang C-C, Wang S-H, Lin C-C, Liu Y-W, et al. Living donor liver transplantation for hepatocellular carcinoma: a single-center experience in Taiwan. Transplantation. United States; 2008;85(3):398-406.

[24] Cherqui D, Laurent A, Alain. Liver resection for transplantable hepatocellular carcinoma. Ann Surg. 2009;250(5). 738-46.

[25] Chan K-M, Yu M-C, Chou H-S, Wu T-J, Lee C-F, Lee W-C. Significance of tumor necrosis for outcome of patients with hepatocellular carcinoma receiving locoregional therapy prior to liver transplantation. Ann Surg Oncol. United States; 2011;18(9):2638-46.

[26] Yoshizumi T, Harimoto N, Itoh S, Okabe H, Kimura K, Uchiyama H, et al. Living donor liver transplantation for hepatocellular carcinoma within milan criteria in the present era. Anticancer Res. Greece; 2016;36(1):439-45. 
[27] Mazzaferro V, Regalia E, Doci R, Andreola S, Pulvirenti A, Bozzetti F, et al. Liver transplantation for the treatment of small hepatocellular carcinomas in patients with cirrhosis. N Engl J Med. United States; 1996;334(11):693-9.

[28] Chan SC, Fan ST. Selection of patients of hepatocellular carcinoma beyond the Milan criteria for liver transplantation. Hepatobiliary Surg Nutr. China (Republic: 1949); 2013;2(2):84-8.

[29] Toso C, Meeberg G, Hernandez-Alejandro R, Dufour J-F, Marotta P, Majno P, et al. Total tumor volume and alpha-fetoprotein for selection of transplant candidates with hepatocellular carcinoma: a prospective validation. Hepatology. United States; 2015;62(1):158-65.

[30] Majno P, Mazzaferro V. Living donor liver transplantation for hepatocellular carcinoma exceeding conventional criteria: questions, answers and demands for a common language. Vol. 12, Liver transplantation: official publication of the American Association for the Study of Liver Diseases and the International Liver Transplantation Society. United States; 2006. p. 896-8.

[31] Kornberg A, Freesmeyer M, Barthel E, Jandt K, Katenkamp K, Steenbeck J, et al. 18FFDG-uptake of hepatocellular carcinoma on PET predicts microvascular tumor invasion in liver transplant patients. Am J Transplant. Denmark; 2009;9(3):592-600.

[32] Hirakawa M, Yoshimitsu K, Irie H, Tajima T, Nishie A, Asayama Y, et al. Performance of radiological methods in diagnosing hepatocellular carcinoma preoperatively in a recipient of living related liver transplantation: comparison with step section histopathology. Jpn J Radiol. Japan; 2011;29(2):129-37.

[33] Piardi T, Gheza F, Ellero B, Woehl-Jaegle ML, Ntourakis D, Cantu M, et al. Number and Tumor Size Are Not Sufficient Criteria to Select Patients for Liver Transplantation for Hepatocellular Carcinoma. Ann Surg Oncol. 2012;19(6):2020-6.

[34] Na GH, Kim DG, Han JH, Kim EY, Lee SH, Hong TH, et al. Inflammatory markers as selection criteria of hepatocellular carcinoma in living-donor liver transplantation. World J Gastroenterol. United States; 2014;20(21):6594-601.

[35] Hong G, Suh K-S, Suh S-W, Yoo T, Kim H, Park M-S, et al. Alpha-fetoprotein and (18)FFDG positron emission tomography predict tumor recurrence better than Milan criteria in living donor liver transplantation. J Hepatol. Netherlands; 2016;64(4):852-9.

[36] Ito T, Takada Y, Ueda M, Haga H, Maetani Y, Oike F, et al. Expansion of selection criteria for patients with hepatocellular carcinoma in living donor liver transplantation. Liver Transpl. United States; 2007;13(12):1637-44.

[37] Yao FY, Ferrell L, Bass NM, Watson JJ, Bacchetti P, Venook A, et al. Liver transplantation for hepatocellular carcinoma: expansion of the tumor size limits does not adversely impact survival. Hepatology. United States; 2001;33(6):1394-403. 
[38] Lee S-G, Hwang S, Moon D-B, Ahn C-S, Kim K-H, Sung K-B, et al. Expanded indication criteria of living donor liver transplantation for hepatocellular carcinoma at one largevolume center. Liver Transpl. United States; 2008;14(7):935-45.

[39] Zheng S-S, Xu X, Wu J, Chen J, Wang W-L, Zhang M, et al. Liver transplantation for hepatocellular carcinoma: Hangzhou experiences. Transplantation. United States; 2008;85(12):1726-32.

[40] Sugawara Y, Tamura S, Makuuchi M. Living donor liver transplantation for hepatocellular carcinoma: Tokyo University series. Dig Dis. Switzerland; 2007;25(4):310-2.

[41] Mazzaferro V, Llovet JM, Miceli R, Bhoori S, Schiavo M, Mariani L, et al. Predicting survival after liver transplantation in patients with hepatocellular carcinoma beyond the Milan criteria: a retrospective, exploratory analysis. Lancet Oncol. 2009;10(1):35-43. Available from: http://dx.doi.org/10.1016/S1470-2045(08)70284-5

[42] Taketomi A, Sanefuji K, Soejima Y, Yoshizumi T, Uhciyama H, Ikegami T, et al. Impact of des-gamma-carboxy prothrombin and tumor size on the recurrence of hepatocellular carcinoma after living donor liver transplantation. Transplantation. United States; 2009;87(4):531-7.

[43] Takada Y, Uemoto S. Liver transplantation for hepatocellular carcinoma: the Kyoto experience. J Hepatobiliary Pancreat Sci. Japan; 2010;17(5):527-32.

[44] DuBay D, Sandroussi C, Sandhu L, Cleary S, Guba M, Cattral MS, et al. Liver transplantation for advanced hepatocellular carcinoma using poor tumor differentiation on biopsy as an exclusion criterion. Ann Surg. United States; 2011;253(1):166-72.

[45] Yoshizumi T, Ikegami T, Toshima T, Harimoto N, Uchiyama H, Soejima Y, et al. Twostep selection criteria for living donor liver transplantation in patients with hepatocellular carcinoma. Transplant Proc. United States; 2013;45(9):3310-3.

[46] Yao FY, Xiao L, Bass NM, Kerlan R, Ascher NL, Roberts JP. Liver transplantation for hepatocellular carcinoma: validation of the UCSF-expanded criteria based on preoperative imaging. Am J Transplant. 2007;7(11):2587-96.

[47] Toso C, Asthana S, Bigam DL, Shapiro AMJ, Kneteman NM. Reassessing selection criteria prior to liver transplantation for hepatocellular carcinoma utilizing the scientific registry of transplant recipients database. Hepatology. 2009;49(3):832-8.

[48] Yang SH, Suh KS, Lee HW, Cho EH, Cho JY, Cho YB, et al. A revised scoring system utilizing serum alphafetoprotein levels to expand candidates for living donor transplantation in hepatocellular carcinoma. Surgery. 2007;141(5):598-609.

[49] Lu C-H, Chen C-L, Cheng Y-F, Huang T-L, Tsang LL-C, Ou H-Y, et al. Correlation between imaging and pathologic findings in explanted livers of hepatocellular carcinoma cases. Transplant Proc. United States; 2010;42(3):830-3. 
[50] Wu Y-J, Lin C-C, Lin Y-H, Wang S-H, Lin T-L, Chen C-L, et al. Incidentally small pulmonary nodule in candidates for living donor liver transplantation. Ann Transplant. Poland; 2015;20:734-40.

[51] Hameed B, Mehta N, Sapisochin G, Roberts JP, Yao FY. Alpha-fetoprotein level> 1000 $\mathrm{ng} / \mathrm{mL}$ as an exclusion criterion for liver transplantation in patients with hepatocellular carcinoma meeting the milan criteria. Liver Transpl. 2014;20(8):945-51.

[52] Chiu KW, Nakano T, Chen KD, Hsu LW, Lai CY, Huang CY, et al. Repeated-measures implication of hepatocellular carcinoma biomarkers in living donor liver transplantation. PLoS One. 2015;10(5):1-12. Available from: <Go to ISI >://WOS:000354916100020

[53] Hsu C-C, Chen C-L, Wang C-C, Lin C-C, Yong C-C, Wang S-H, et al. Combination of FDG-PET and UCSF criteria for predicting HCC recurrence after living donor liver transplantation. Transplantat. 2016 Sep;100(9):1925-32.

[54] Orci LA, Berney T, Majno PE, Lacotte S, Oldani G, Morel P, et al. Donor characteristics and risk of hepatocellular carcinoma recurrence after liver transplantation. Br J Surg. England; 2015;102(10):1250-7.

[55] Park G-C, Song G-W, Moon D-B, Lee S-G. A review of current status of living donor liver transplantation. Hepatobiliary Surg Nutr. China (Republic: 1949); 2016;5(2):10717.

[56] Toso C, Mentha G, Kneteman NM, Majno P. The place of downstaging for hepatocellular carcinoma. J Hepatol. 2010;52(6):930-6. Available from: http://dx.doi.org/10.1016/ j.jhep.2009.12.032

[57] Yu CY, Ou HY, Huang TL, Chen TY, Tsang LLC, Chen CL, et al. Hepatocellular carcinoma downstaging in liver transplantation. Transplant Proc. 2012;44(2):412-4.

[58] Na GH, Kim EY, Hong TH, You YK, Kim DG. Effects of loco regional treatments before living donor liver transplantation on overall survival and recurrence-free survival in South Korean patients with hepatocellular carcinoma. HPB (Oxford). England; 2016;18(1):98-106.

[59] Parikh ND, Waljee AK, Singal AG. Downstaging hepatocellular carcinoma: a systematic review and pooled analysis. Liver Transplant. 2015;21(9):1142-52. Available from: http://www.ncbi.nlm.nih.gov/pubmed/25981135 [cited 2015 Nov 17].

[60] Xu D-W, Wan P, Xia Q. Liver transplantation for hepatocellular carcinoma beyond the Milan criteria: a review. World J Gastroenterol. United States; 2016;22(12):3325-34.

[61] Majeed TA, Wai CT, Rajekar H, Lee KH, Wong SY, Leong SO, et al. Experience of the transplant team is an important factor for posttransplant survival in patients with hepatocellular carcinoma undergoing living-donor liver transplantation. Transplant Proc. United States; 2008;40(8):2507-9. 
[62] Chen C-L, Concejero AM. Early post-operative complications in living donor liver transplantation: prevention, detection and management. HBPD INT. China; 2007;6(4): 345-7.

[63] Chen C-L, Concejero AM, Cheng Y-F. More than a quarter of a century of liver transplantation in Kaohsiung Chang Gung Memorial Hospital. Clin Transpl. United States; 2011;213-21.

[64] Jawan B, Wang C-H, Chen C-L, Huang C-J, Cheng K-W, Wu S-C, et al. Review of anesthesia in liver transplantation. Acta Anaesthesiol Taiwanica. Taiwan LLC; 2014;52(4):185-96. Available from: http://linkinghub.elsevier.com/retrieve/pii/ S1875459714001088

[65] Nadalin, S., Bockhorn, M., Malagó, M., Valentin-Gamazo, C., Frilling, A., \& Broelsch, C. E. (2006). Living donor liver transplantation. $H P B: 8(1), 10-21$. http://doi.org/ $10.1080 / 13651820500465626$.

[66] Abdeldayem HM, editor. Living Donor Liver Transplantation, Liver Transplantation Technical Issues and Complications. InTech. 2012. Available from: http://www.intechopen.com/books/liver-transplantation-technical-issues-and-complications/livingdonor-livertransplantation

[67] Ercolani G, Grazi GL, Ravaioli M, Grigioni WF, Cescon M, Gardini A, et al. The role of lymphadenectomy for liver tumors. Ann Surg. 2004;239(2):202-9.

[68] Sotiropoulos GC, Malago M, Molmenti EP, Losch C, Lang H, Frilling A, et al. Hilar lymph nodes sampling at the time of liver transplantation for hepatocellular carcinoma: to do or not to do? Meta-analysis to determine the impact of hilar lymph nodes metastases on tumor recurrence and survival in patients with hepatocellular carcin. Transpl Int. 2007;20(2):141-6. Available from: http://www.ncbi.nlm.nih.gov/pubmed/ 17239022\nhttp://onlinelibrary.wiley.com/doi/10.1111/j.1432-2277.2006.00412.x/ abstract?systemMessage $=$ Wiley + Online + Library+will+be + disrupted + on $+7+J u l y+$ from +10:00-12:00+BST+(05:00-07:00+EDT)+for+essential+maintenance

[69] Suh S-W, Lee J-M, You T, Choi YR, Yi N-J, Lee K-W, et al. Hepatic venous congestion in living donor grafts in liver transplantation: is there an effect on hepatocellular carcinoma recurrence? Liver Transplant Off Publ Am Assoc Study Liver Dis Int Liver Transplant Soc. United States; 2014;20(7):784-90.

[70] Matsuda H, Sadamori H, Shinoura S, Umeda Y, Yoshida R, Satoh D, et al. Aggressive combined resection of hepatic inferior vena cava, with replacement by a ringed expanded polytetrafluoroethylene graft, in living-donor liver transplantation for hepatocellular carcinoma beyond the Milan criteria. J Hepatobiliary Pancreat Sci. Japan; 2010;17(5):719-24.

[71] Pahari H, Li W-F, Lin T-S, Wang C-C, Yong C-C, Lin T-L, et al. Extensive adhesions in living donor liver transplantation: a retrospective analysis. World J Surg. 2015;40(2): 427-32. Available from: http://link.springer.com/10.1007/s00268-015-3219-x 
[72] Memeo R, De'Angelis N, de Blasi V, Cherkaoui Z, Brunetti O, Longo V, et al. Innovative surgical approaches for hepatocellular carcinoma. World J Hepatol. United States; 2016;8(13):591-6.

[73] Lin T-S, Chiang Y-C, Chen C-L, Concejero AM, Cheng Y-F, Wang C-C, et al. Intimal dissection of the hepatic artery following transarterial embolization for hepatocellular carcinoma: an intraoperative problem in adult living donor liver transplantation. Liver Transplant Off Publ Am Assoc Study Liver Dis Int Liver Transplant Soc. United States; 2009;15(11):1553-6.

[74] Pomfret EA, Lodge JPA, Villamil FG, Siegler M. Should we use living donor grafts for patients with hepatocellular carcinoma? Ethical considerations. Liver Transplant Off Publ Am Assoc Study Liver Dis Int Liver Transplant Soc. United States; 2011;17 Suppl 2:S128-32.

[75] Mizuno S, Yokoi H, Shiraki K, Usui M, Sakurai H, Tabata M, et al. Prospective study on the outcome of patients with hepatocellular carcinoma registered for living donor liver transplantation: how long can they wait? Transplantat. United States; 2010;89(6): $650-4$.

[76] Taefi a, Abrishami a, Eghtesad B, Sherman M. Surgical resection versus liver transplant for patients with hepatocellular carcinoma (Review). Cochrane Database Syst Rev. 2013; (6).

[77] Ho C, Lee P, Chen C, Ho M. Long-Term outcomes after resection versus transplantation for hepatocellular carcinoma within UCSF criteria. Ann Surg Oncol. 2012;19(3):826-33.

[78] Dai WC, Chan SC, Chok KSH, Cheung TT, Sharr WW, Chan ACY, et al. Good longterm survival after primary living donor liver transplantation for solitary hepatocellular carcinomas up to $8 \mathrm{~cm}$ in diameter. HPB (Oxford). England; 2014;16(8):749-57.

[79] Blumgart LH, Jarnagin WR, Belghiti J, Buchler MW, Chapman WC, M.I. D, et al., editors. Liver Transplantation: Indications and General Considerations. In: Blumgart's Surgery of the Liver, Biliary Tract, and Pancreas. 5th ed. Philadelphia: Elsevier; 2012.

[80] Silva MF, Sapisochin G, Strasser SI, Hewa-geeganage S, Chen J, Wigg AJ. Liver resection and transplantation offer similar 5-year survival for Child-Pugh-Turcotte A HCC-patients with a single nodule up to $5 \mathrm{~cm}$ : a multicenter, exploratory analysis. Eur J Surg Oncol. 2013;39(4):386-95. Available from: http://dx.doi.org/ 10.1016/j.ejso.2012.12.011

[81] Bruix J, Sherman M. Management of hepatocellular carcinoma: an update. Hepatol. United States; 2011;53(3):1020-2.

[82] Sapisochin G, Castells L, Dopazo C. Single HCC in cirrhotic patients: liver resection or liver transplantation?? Long-term outcome according to an intention-to-treat basis. Ann Surg Oncol. 2013;20(4):1194-202. 
[83] Dhir M, Lyden ER, Smith LM, Are C. Comparison of outcomes of transplantation and resection in patients with early hepatocellular carcinoma: a meta-analysis. Hpb. 2012;14(9):635-45.

[84] Proneth A, Zeman F, Schlitt HJ, Schnitzbauer A. Is resection or transplantation the ideal treatment in patients with hepatocellular carcinoma in cirrhosis if both are possible? A systematic review and metaanalysis. Ann Surg Oncol. 2014;21(9):3096-107. Available from: http://www.ncbi.nlm.nih.gov/pubmed/24866437

[85] Kaido T, Morita S, Tanaka S, Ogawa K, Mori A, Hatano E, et al. Long-term outcomes of hepatic resection versus living donor liver transplantation for hepatocellular carcinoma: a propensity score-matching study. Dis Markers. United States; 2015;2015:425926.

[86] Dionigi R. One Liver for Two: Split and Living Donor Liver Transplantation for Adult and Pediatric Patients. In: Recent Advances in Liver Surgery. Austin, TX: Landes Bioscience; 2009.

[87] Di Sandro S, Slim AO, Giacomoni A, Lauterio A, Mangoni I, Aseni P, et al. Living donor liver transplantation for hepatocellular carcinoma: long-term results compared with deceased donor liver transplantation. Transplant Proc. United States; 2009;41(4):12835 .

[88] Lee S-G, Moon D-B, Shin H, Kim K-H, Ahn C-S, Ha T-Y, et al. Living donor liver transplantation for hepatocellular carcinoma: current status in Korea. Transplant Proc. United States; 2012;44(2):520-2.

[89] Hu Z, Qian Z, Wu J, Zhou J, Zhang M, Zhou L, et al. Clinical outcomes and risk factors of hepatocellular carcinoma treated by liver transplantation: a multi-centre comparison of living donor and deceased donor transplantation. Clin Res Hepatol Gastroenterol. 2015; 2016 Jun;40(3):315-26

[90] Fisher RA, Kulik LM, Freise CE, Lok ASF, Shearon TH, Brown RSJ, et al. Hepatocellular carcinoma recurrence and death following living and deceased donor liver transplantation. Am J Transplant. Denmark; 2007;7(6):1601-8.

[91] Park M-S, Lee K-W, Suh S-W, You T, Choi Y, Kim H, et al. Living-donor liver transplantation associated with higher incidence of hepatocellular carcinoma recurrence than deceased-donor liver transplantation. Transplant J. 2014;97(1):71-7. Available from: http://content.wkhealth.com/linkback/openurl?sid=WKPTLP:landingpage\&an= 00007890-201401150-00012

[92] Ninomiya M, Shirabe K, Facciuto ME, Schwartz ME, Florman SS, Yoshizumi T, et al. Comparative study of living and deceased donor liver transplantation as a treatment for hepatocellular carcinoma. J Am Coll Surg. United States; 2015;220(3):297-304.e3. 